\title{
E se il telefono non suona più?
}

In questi giorni Francesco Varanini su Bloom, frammenti di organizzazione (www.bloom.it),ha pubblicato i risultati di una ricerca svolta nel corso del 2012 dal titolo: "Artigiani e Piccoli Imprenditori come persone. Le conseguenze della crisi 2007-2012 sugli atteggiamenti e sulle motivazioni”.

La lettura di questo report costruito in forma quali-quantitativa ha suggerito il tema di questo editoriale almeno per due motivi. Il primo perché consente di percepire e cogliere la natura delle emozioni avvertite da chi si trova a vivere momenti di intensa discontinuità nelle proprie usuali prospettive; il secondo perché la sua lettura ripropone perentoriamente agli studiosi d'impresa una riflessione profonda sul proprio ruolo nel sistema socio-economico, soprattutto in momenti di crisi come quello che stiamo attraversando.

La natura della prima sollecitazione si coglie facilmente riprendendo alcune affermazioni degli intervistati contenute nel report citato e tratte dalle venti interviste in profondità condotte con gli imprenditori coinvolti dello studio. La percezione si completa con il riferimento ad alcune misurazioni quantitative, riferite alle frequenze registrate per le risposte ad alcune domande proposte in una seconda fase dello studio sulla base di un questionario predefinito.

La seconda sollecitazione scaturisce immediatamente dalla semplice lettura dei risultati della ricerca. Diviene per così dire automatica, perché al di là degli sforzi per conseguire il rigor richiesto per la pubblicazione nei journal di management induce a mettere in discussione il senso ultimo dell'attività degli studiosi di impresa.

Ovviamente, in questa sede non potremo che prendere in considerazione una selezione delle informazioni raccolte nella ricerca citata. E lo faremo in forme raccordate cercando di connettere più affermazioni a sé stanti ma partecipi dello stesso tema indagato.

Siamo ben consapevoli che ogni volta che si propone una selezione si tracciano delle linee di demarcazione del fenomeno indagato frutto del sentire di chi le traccia. Va quindi precisato - in particolare perché il tema investe le emozioni - che, oltre a non essere esaustiva, la nostra analisi potrebbe anche risultare di parte perché difficilmente può prescindere da giudizi di valore che portiamo con noi stessi quando si trattano temi come questo. Temi che muovono il pensiero secondo logiche razionali ma anche non razionali ed emotive.

Per questi motivi invitiamo i lettori interessati, e crediamo che gli studiosi di impresa potrebbero far parte di tale gruppo, a scorrere l'intera ricerca per cogliere appieno la natura del fenomeno indagato attraverso le proprie lenti di lettura ed interpretazione della realtà, attraverso, cioè, il proprio modo di sentire e di vedere.

Lo studio si avvia secondo le regole dello story telling, quindi sulla base della raccolta di pensieri espressi dai testimoni privilegiati che hanno partecipato allo studio.

sinergie, rivista di studi e ricerche

n. 91, Maggio-Agosto 2013, pp. VII-XII

ISSN 0393-5108 - DOI 10.7433/s91.2013.01 
Pensieri intercalati da brevi commenti dell'autore della ricerca e raccolti in una serie di punti: il mestiere e il lavoro, l'uso de tempo, l'atteggiamento, la lettura della crisi e il senso dell'andare avanti.

"Mi piaceva fare le cose con le mani, lasciare traccia", "la perfezione nell'artigianato deve avere irregolarità codificate", "l'imperfezione manuale è ovvia".

In queste brevi affermazioni si coglie tutta la natura del Mestiere e la sua diversità rispetto ad un lavoro che tale non sia. Il mestiere è a tutto tondo, chi lo padroneggia dispone di un sapere fare le cose dall'inizio alla fine, dalla progettazione alla realizzazione in una manualità accentuata anche se mediata da moderne strumentazioni tecnologiche. Per questo il mestiere conduce a piccole imperfezioni, che fanno parte di questo modo di procedere che non replica, non omologa, lascia traccia di sé nel trasmettere un'anima in ciò che si fa, attraverso un'arte capace di rispondere in modo personalizzato ed unico a chi propone un problema all'impresa.

"Lavorare non mi pesa. Non ho orari. Quando devo finire un lavoro, specialmente quando sto per concluderlo, continuo a lavorare la sera fino a tardi".

$\mathrm{Si}$ avverte in questa affermazione il profondo senso di piacere provato nello svolgere il proprio lavoro. Il lavoro non pesa, certamente è faticoso e stancante ma viene avvertito come "giocoso", "dilettevole", al punto tale che non esiste una netta separazione tra tempo di lavoro e tempo libero. Si tratta, infatti di una distinzione che interessa a chi avverte il lavoro come un sacrificio, un qualcosa da fare, per ottenerne un reddito sufficiente a soddisfare le esigenze di sé e della famiglia, non interessa a chi nel lavoro in qualche modo si diverte.

Il pensiero corre immediato al Primo Levi della Chiave a stella, romanzo nel quale l'A. ricorda che "l'amore per il proprio lavoro, che è privilegio di pochi, costituisce la più grande approssimazione concreta alla felicità sulla terra”.

“Quando c’è la passione non c'è fatica”, "tutti i giorni cerchi qualcosa”.

In questo dire è raccolto un profondo sentimento di entusiasmo per quello che si fa, per un qualcosa che appare come il lavoro più bello del mondo, per un qualcosa di cui si è innamorati, come il primo giorno in cui si è avviata l'avventura imprenditoriale.

È questo un sentimento tanto prezioso quanto poco diffuso in un mondo del lavoro frequentemente spersonalizzato, nel quale a volte le persone non sanno nemmeno bene come si collochi la propria parcellizzata azione all'interno del processo produttivo d'impresa. Sentimento che rappresenta un immenso tesoro da preservare e diffondere anche nelle imprese industriali, i cui processi non si sviluppano per mestieri ma per micro fasi omogenee di lavorazione che 
difficilmente riescono a valorizzare le energie che questo stato dell'animo consente di movimentare.

Fatte queste premesse sui tratti del modello d'impresa indagato lo studio entra nell'analisi della crisi e qui i toni si fanno decisamente cupi e preoccupati seppur incapaci di riflettere una rassegnazione senza via di uscita.

"Stiamo vedendo e vivendo una crisi senza precedenti", "il clima si avvelena", "un muro dappertutto", "sono costretto a vivere da sconfitto eppure non è per causa mia che si è creata questa situazione".

Il mondo è cambiato, come sempre peraltro è accaduto. Ma questa volta è cambiato con una intensità ed una velocità tali da far avvertire all'imprenditore come un senso di accerchiamento, difficile da decodificare nella sua natura di fondo e come tale fonte di disorientamento. A ciò contribuisce la difficoltà di intravvedere la strada nuova da percorrere, magari solo confusamente intravista attraverso una cassetta degli attrezzi gestionali inadatta a focalizzare il significato vero della drammatica caduta di domanda, soprattutto interna, che si avverte. Il presente sembra distaccarsi completamente dal passato, non consentendo di pensare ad un futuro da costruire perché non si riesce ad immaginarlo. Così la crisi diventa anche crisi di valori e personale.

"Non è solo che manca equità, proprio non ci sono regole", "ci sono molti che ne approfittano e abbassano l'asticella etica", "abbiamo direttori di banca che insultano le famiglie", "se qualcuno ci dicesse qualcosa continuerei a fare con più tranquillità quello che faccio", "abbiamo bisogno di essere educati, di avere informazioni”.

Un senso di smarrimento e di abbandono, oltre che di emarginazione, traspaiono dalla semplice lettura di queste ultime affermazioni. Tuttavia non viene meno il senso morale che sa riconoscere i comportamenti scorretti. Sembra che il quadro delle regole sia venuto meno anche nella confusione che si è impadronita dei sistemi costruttori di regole, a loro volta travolti dal vortice della crisi e incapaci di tracciare una rotta all'interno di una tempesta che richiederebbe -come nel Tifone di Conrad il coraggio del capitano MacWhirr di andare contro le regole fissate nei manuali per fronteggiare il tifone che la nave si trovava ad affrontare. Il coraggio di produrre scelte diverse e controcorrente ma guidate dall'assunzione di una responsabilità che oggi sembra destinata a rimanere orfana, destinata a non trovare persone disponibili ad accoglierla, in un vuoto discorrere produttore di una sinergia negativa che attraverso ansia e frustrazione facilmente potrebbe aprire le porte alla depressione e alla disperazione.

Puntuale, peraltro, è la richiesta di educazione in termini di formazione e informazione, come emerge anche da altre affermazioni che sostengono la necessità di una formazione continua, oltre che di una formazione d'ingresso nell'attività. 
Aspetto, questo, che sottolinea il riconoscimento dell'inadeguatezza delle conoscenze possedute, incapaci di leggere il divenire in atto per tradurlo in una rivisitazione del proprio modo d'essere per poter partecipare alla costruzione del futuro.

"Se il telefono non suona è terribile", "sono anestetizzato, sono in stand by", "avendo perso l'entusiasmo l'umore è peggiorato tantissimo", "ho vissuto questa esperienza in famiglia, mio padre è fallito, lui occultava tutto per vergogna", "in casa è diventata una atmosfera invivibile", "non ho niente da far fare a chi lavora con me", "devo mettere in cassa integrazione, devo licenziare".

Il venir meno del lavoro, che rappresentava parte integrante della vita della persona e che forniva anche un riconoscimento sociale per il valore riconosciuto al mestiere ed alla capacità di lavorare e di dar da lavorare, espelle l'entusiasmo e l'euforia per accogliere l'ansia, la paura e la frustrazione con il timore di perdere la dignità derivante dall'essere parte attiva di una comunità.

Lo stand by richiamato potrebbe invero diventare una trappola dalla quale difficilmente si può uscire, perché se i tempi di ozio creativo sono fondamentali nei momenti di intensa attività, $i$ tempi di ozio forzato possono avvolgere la persona in una spirale di emozioni negative tali da indurla a non cercare alternative al "telefono che non suona", in una vana attesa di un qualcosa che potrebbe non arrivare mai.

Nella fase quantitativa, condotta attraverso 185 interviste on line, lo stato d'animo prevalentemente segnalato dagli intervistati vede questa classifica:

1) Speranza, $67 \%$

2) Rabbia $58,9 \%$

3) Ansia $56,8 \%$

4) Frustrazione $31,9 \%$

5) Fiducia $17,3 \%$

6) Umiliazione $8,6 \%$

7) Vergogna $2,2 \%$

Tra i sentimenti proposti nell'intervista è importante notare come quello che raccoglie la maggioranza dei consensi- ogni intervistato poteva segnalare al massimo 3 tra i 7 sentimenti proposti - sia la speranza, quindi un sentimento positivo. Speranza probabilmente legata alla certezza del valore del mestiere e quindi alla volontà di agire per riaffermarlo nella nuova situazione che si è venuta a creare. Speranza che non rappresenta una fiduciosa attesa in un qualcosa che verrà dall'alto da parte di qualche altro sistema, quanto il desiderio di riproporsi attivamente per la creazione del futuro, una speranza attiva come la definiva Ernst Bloch. Speranza, però, che per diventare tale necessita di un sostegno nella decodificazione della complessità del problema, per muovere di conseguenza nelle nuove direzioni che potrebbero emergere da una rinnovata capacità di inoltrarsi nel folto bosco delle idee nuove che potrebbero scaturire dalla rivisitazione delle conoscenza disponibili. 
Rilevante è anche la segnalazione del valore, anche se ben più ridotto, assegnato alla fiducia, quindi al senso di affidamento nelle relazioni, riconoscimento che non è venuto meno pur nel burrascoso periodo in atto.

Invero, i sentimenti negativi segnalati fanno pensare più ad un senso di impotenza che ad un senso di rinuncia ad un proprio ruolo nella società.

In realtà, rispondendo alla domanda su quali sensazioni provino, gli intervistati mettono ai primi 4 posti le seguenti affermazioni:

"È dura lavorare senza soddisfazione", 48,6\%

"Mi rifiuto di cedere", 46,5\%

"Stiamo sopravvivendo, non vivendo", 44,3\%

"Questa situazione è una sfida e la voglio vincere”, 40,5\%

risposte che alternano sconforto a speranza attiva, a volontà cioè di uscire dalla situazione che si è determinata, aspetto questo che in qualche modo si può ritenere prevalente se si considera che il 51,9\% degli intervistati ritiene che questa crisi possa consentire di riscoprire l'importanza dell'artigianato e della piccola impresa.

"Se questa cosa non funziona pazienza, ne proverò un'altra”, "non si può starsene chiusi nelle proprie quattro mura ad aspettare che il telefono suoni, bisogna viaggiare all'estero", "il problema dell'artigiano è che non ha un banalissimo sito internet, è una bomba se usato bene", "mollare no, non mollo, vado all'estero da un'altra parte", stare nel proprio buco non è fruttuoso, la comunicazione tra artigiani è basilare", "rischiamo di perdere il nostro vero patrimonio: capacità e stile artigianale", "la crisi tira fuori il meglio di noi, ci costringe a darci da fare”.

Due strade paiono emergere da questo gruppo di affermazioni: l'una l'apertura al mondo, l'altra la coltivazione dell'unicità dello stile e della bellezza del prodotto artigiano. Strade che però potrebbero ben fondersi ricorrendo all'aiuto dei moderni sistemi di comunicazione che possono facilmente veicolare nel mondo la bellezza di un prodotto plasmato dalle mani di un "maestro artigiano", un prodotto che possiede un'anima e che sa dialogare con chi lo produce e con chi lo usa.

Due ulteriori affermazioni riportate nello studio meritano di non passare inosservate:

"Non dimenticare la dimensione umana, siamo persone, non numeri";

"Un buon artigiano deve leggere libri, un buon intellettuale deve lavare i piatti, è una cosa che fa bene".

La prima ci pone dinnanzi alla brutalità con la quale i mass media trattano del problema della crisi delle piccole imprese, pressoché ignorate in favore degli ampi spazi dedicati al vacuo dibattito politico popolato da discorsi pieni di vuoto dove il burocratese è divenuto ormai norma. Gli unici spazi, piccoli quanto basta, vanno a sottolineare i casi di suicidio di un imprenditore o di lavoratori rimasti senza lavoro, perché questo fa notizia. Mai uno spazio dedicato alla tristezza ed alla tragedia che la fabbrica chiusa, popolata di erbacce che gradualmente la seppelliscono, diffonde 
nella comunità e negli ignari passanti, quando prima era viva e popolata di persone che si muovevano nei loro ruoli, in un rincorrersi quasi musicale di rumori che ora hanno lasciato spazio ad uno spaventoso e assordante silenzio.

Le persone sono diventate dei semplici numeri, come quelli delle quotazioni di borsa dietro cui si nasconde quella finanza egoistica che ha portato il sistema capitalistico sull'orlo della sua fine a causa dell'insopportabilità sociale delle sperequazioni che ha generato.

Ma le persone sono persone, ovviamente anche numeri per la statistica e la ricerca, ma mai numeri nei sentimenti e nelle emozioni provate.

Invero, se il buon artigiano deve leggere libri, è certamente vero che l'intellettuale deve lavare i piatti, e noi, studiosi d'impresa apparteniamo a questa categoria.

Quei piatti non sono che un richiamo al fatto che l'intellettuale, lo studioso d'impresa nel nostro caso, non deve muoversi in un mondo che sia separato dal reale.

Intendiamoci, l'immaginazione, la fantasia, la creatività, la curiosità, l'esplorazione di strade che apparentemente non vanno in nessun posto, l'andare controcorrente, la ricerca dell'impossibile restano parte integrante del lavoro del ricercatore.

Ma la finalità che lo muove nel suo desiderio di conoscenza non può che essere il riferirsi all'impresa, alla sua vita, alla sua morte, al suo divenire, alla sua capacità di vincere il tempo come accade per le imprese ultracentenarie, alle emozioni di chi la vive, alle relazioni sociali che sostiene, alla qualità della vita che contribuisce a diffondere, ai processi decisionali che la caratterizzano ed alle forme organizzative e alle tecniche che la popolano. E tutto questo con lo sguardo alzato al futuro, vivendo il presente nella memoria di un passato che consente di innalzare l'edificio della conoscenza per fare dell'impresa un posto bello dove lavorare e un produttore e diffusore di progresso.

Quanto di tutto questo la nostra comunità sta facendo? Quanto tempo dedica a costruire ponti tra il pensiero e l'azione e quanto alla sfida dell'impact factor in una moderna e sterile autoreferenzialità agghindata nei suntuosi abiti della double blind peer review, ma spesso rattrappita su esercizi di ginnastica mentale a malapena utili solo a chi li fa?

Non è forse giunto il momento di condurre al nostro interno una riflessione per verificare come poter portare la capacità di produrre idee ed innovazione di cui disponiamo a sostegno del progresso del nostro Paese e delle imprese che lo popolano?

O forse ci illudiamo di farlo già? 